\title{
Figure S11
}

\author{
L. major \\ L. tropica \\ L. donovani \\ L. infantum
}

L. major

L. tropica

L. donovani

L. infantum

L. major

L. tropica

L. donovani

L. infantum

L. major

L. tropica

L. donovani

L. infantum

L. major

L. tropica

L. donovani

L. infantum

L. major

L. tropica

L. donovani

L. infantum

L. major

L. tropica

L. donovani

L. infantum

L. major

L. tropica

L. donovani

L. infantum

L. major

L. tropica

L. donovani

L. infantum

L. major
L. tropica
L. donovani
L. infantum

L. major

L. tropica
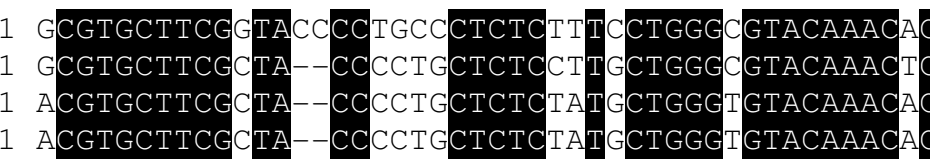

CCACAGCTCGTGCT $T$

CCACAGCTCACGCTG

CCACAGCTCGTGCTG

CCACAGCTCGTGCTG

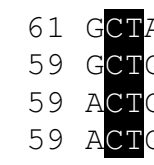

AACCATTTA
GACCATTTA
GACCATTTA
GACCATTTAC

II

11111

II

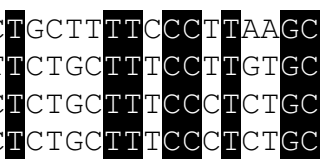

121

118

119

119
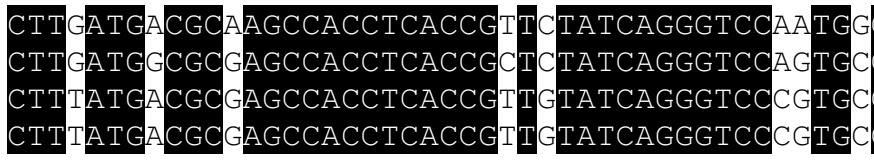

CCACTCTGCGGGAACG
CCACTCTGCGGGAACG
CCACTCTGCGGGAACG
CCACTCTGCGGGAACG
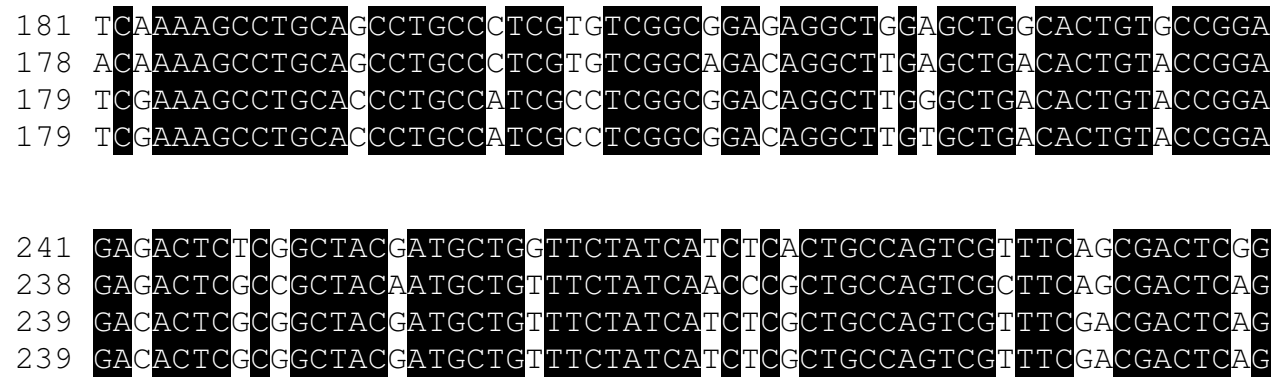

\section{1 \\ 298 \\ 299}

299
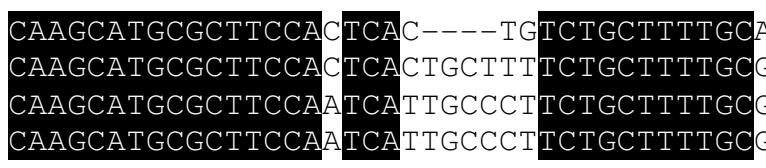

AGCGCTTCGGTAATACCGAGCG
GGCGCTTCGGTAATACCGAGCG
GGCGCTTCGGTAATACCGAGCG
GGCGCTTCGGTAATACCGAGTG
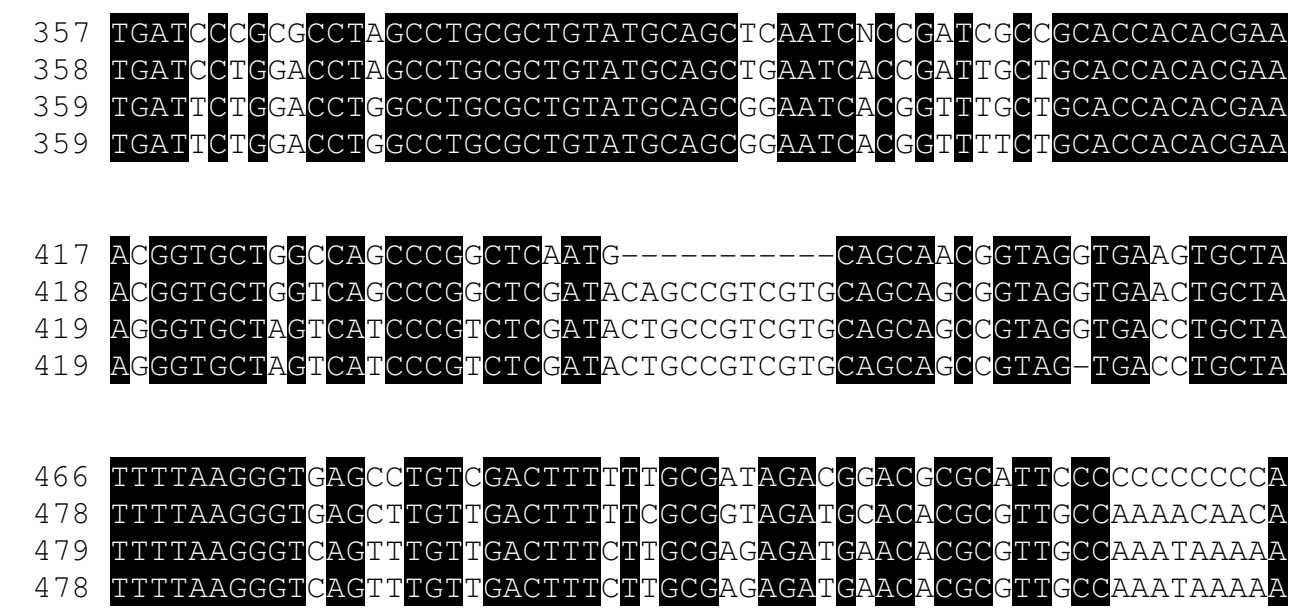

\section{6 \\ 538 \\ 539 AAGAGCATGCTCTCT
538 AAGAGCATGCTCTCT}

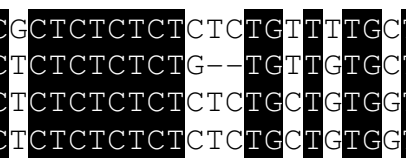

TTCNTGCGCTTTGTGTTGCGCTCTT
CCCTTTGCGTTTGTCTTGCGTTCTT
GCCATTTGCGTTTATGCTGCTTTCTT
ICCATTTGCGTTAATCTGCTTTCTT

586 TATCATCTGC--TITTAGTCCAATGGTGCTCTA
596 ATCCTCTICTTITTCAGTCTGGGTGGTGTCTC

TTTTIGTGCAGAA GGGTAAACTTGTC

596 ATCDTCTICTTTITTCAGTCTGGGTGGTGCTCTCTTTITGGTGCAGAIGGGTGAAACITGT 


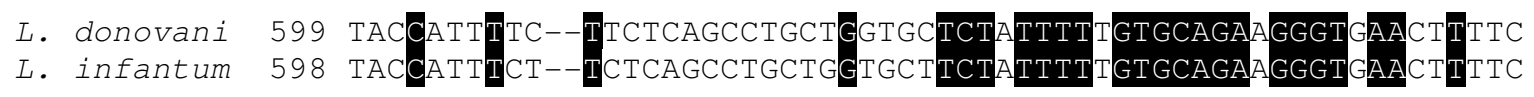

L. major
L. tropica
L. donovani
L. infantum

L. major

L. tropica

L. donovani

L. infantum

L. major

L. tropica

L. donovani

L. infantum

L. major

L. tropica

L. donovani

L. infantum

L. major

L. tropica

L. donovani

L. infantum

L. major

L. tropica

L. donovani

L. infantum
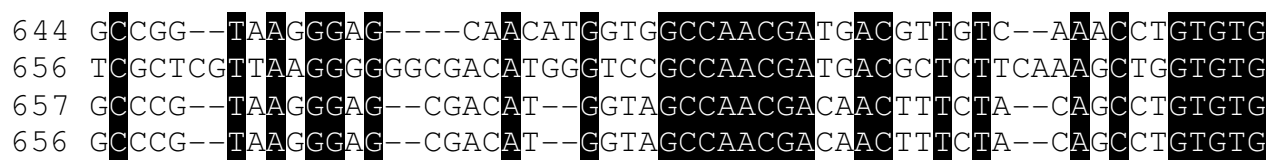

696 ATGTT
716 ATGTT
709 ATGTC
708 ATGTC
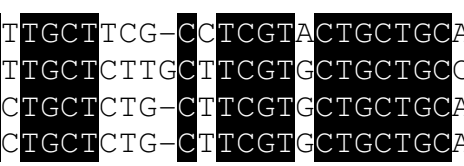

ACTAT
CACTT
АСТT
АCTCT

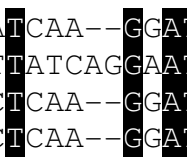

GATACGAAGGCGAATGGGTCTA
AATTACGAAAGGCTACCTCCGG
GATACGGAGGCTATTCGGCCTA
GATACGGAGGCTATTCGGCCTA
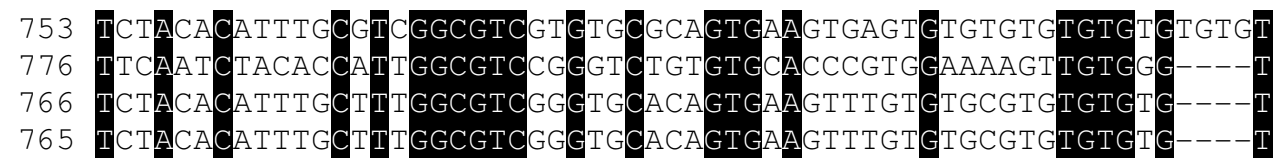

813 GCTGCGAACGATCGATGCGCAT--GACTTTCAAAAGGTGTGCAGGTCTCATCCACAGCAT

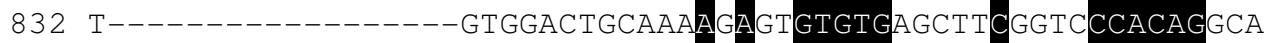
822 GCTGTCAGCAATCAATACGCATACCTTTCAAAAAAGGTGTGCTGGCCTCGTCCACAGCAC 821 GCTGTCAGCAATCAATACGCATACCTTTCAAAAAAGGTGTGCTGGCCTCGTCCACAGCAC

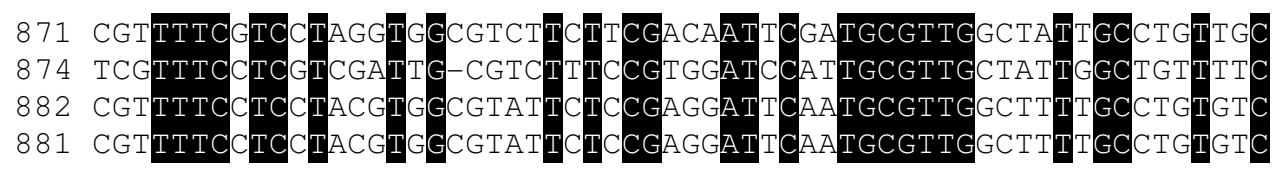

L. major

989

L. tropica 993

L. donovani 1000

L. infantum 999

931 CGTGAAAGTATATAG

933 GTGAAAGTATCTTAG

942 CGCGAAAGTATATATT

941 CGCGAAA
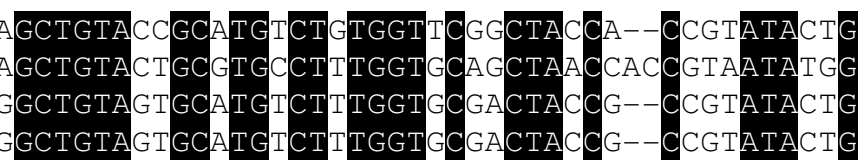

L. major 1046

L. tropica 1053

L. donovani 1060

L. infantum 1059

CTATGt
CTATGT
CTATGC
CTATGC
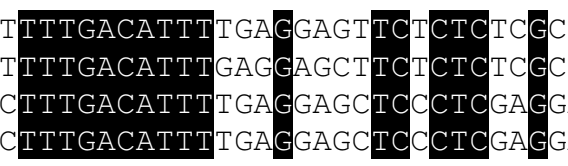

GCGAAAACAA
GCGAAA GAAA
GGAAAAA AAAA

AAA---GCGAAAAAGT GCCG
AAAAGGCGAAACAAGT GCCG
AAGCGAAAAACAAAACGCCG
AAGCGAAAAACAAAACGCCG

$\begin{array}{llll}\text { L. major } & 1106 & \text { GGACTTTCCTTTTTGTT--TCAGCCTTGCTCATATGTGTGATGTTCCTACGTGCTTATCC } \\ \text { L. tropica } & 1113 \text { AGACTTTCCTCTTTTTATTTCAGCCTTGCTCATCTCTATGATGTTCCTACGTGCTTATCT } \\ \text { L. donovani } & 1120 \text { GGGCTTTCCTTTTTTTTC------ATGCTCACCTGTATGATTTCCTCCGTGCTTATCT } \\ \text { L. infantum } & 1119 \text { GGGCTTTCCTTTTTTTTTC------ATGCTCACCTGTATGATTTTCCTCCGTGCTTATCT }\end{array}$

L. major 1164 TTTGAAGTTTCTTTTCTCAGCGCT-------CCTTTCGCTCTTGCGGCTGATAGCACTGA

L. tropica 1173 GTTGAAGTTTTTCTCTTCTCTTTCAGCGCTCTTTTCTCTCTTTGCTGCTGATAGTACTGA

L. donovani 1174 TTTGAATTTCTCTCTTCAGCTCT-------CTTTTTGCTCTTGCTGCTGATAGCGATGG

L. infantum 1173 TTTGAATTTCTCTCTTCAGCTCT-------CTTTTTGCTCTTGCTGCTGATAGCGATGG

L. major 1217 ACAAAACGTTATTGCCGGGAAACCTTTC-CTTTCTGCGTTGTTTTAATATATATATATAT

L. tropica 1233 ATtAAACGTCATtGCTGCAAAATCTTtTTCTTTCTGTGTTGTTCCAAGATGATTATATAT 
L. donovani 1227

L. infantum 1226

L. major 1335

L. tropica 1342 CTTTTTT

L. donovani 1340 TTCTTtCi

L. infantum 1339 TTCTTTCI
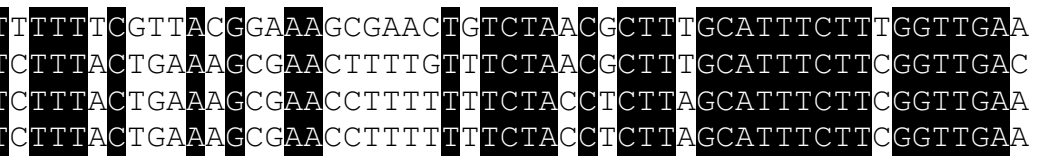

L. major

L. tropica

L. donovani 1400

L. infantum 1399

L. major 1449

L. tropica 1462

L. donovani 1460

L. infantum 1459

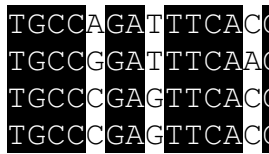

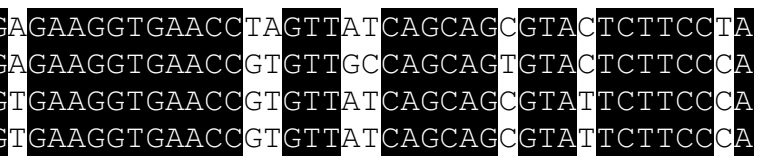
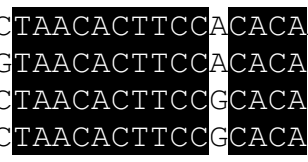

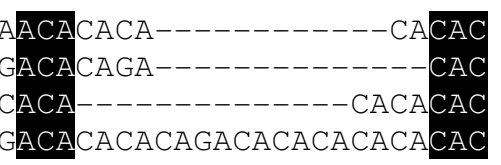

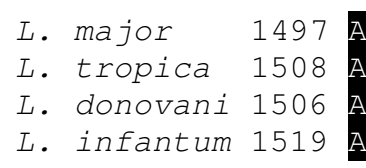

L. major 1557

L. tropica 1568

L. donovani 1566

L. infantum 1579

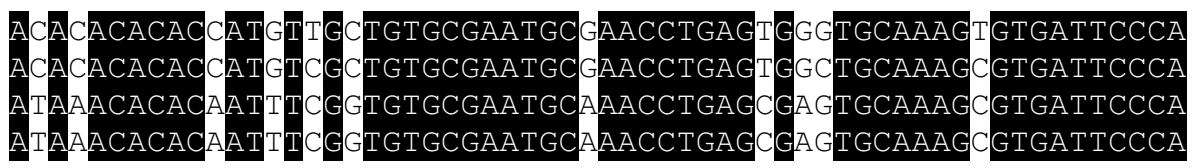

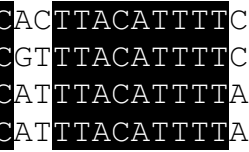

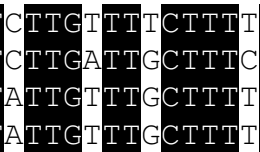

L. major

L. tropica

donovani 1624

L. infantum 1637
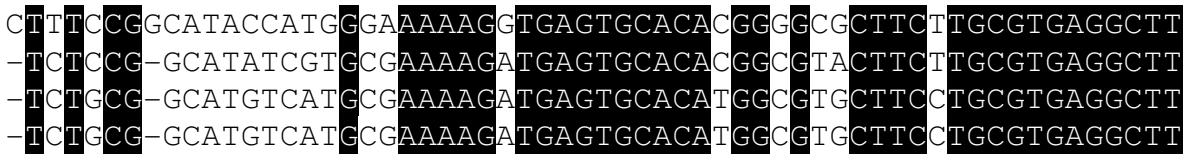

TGAGTGCACA

TGCGTGAGGCT 1

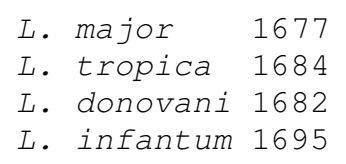

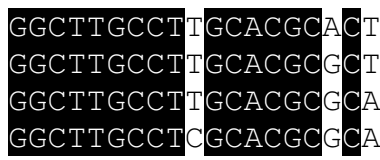

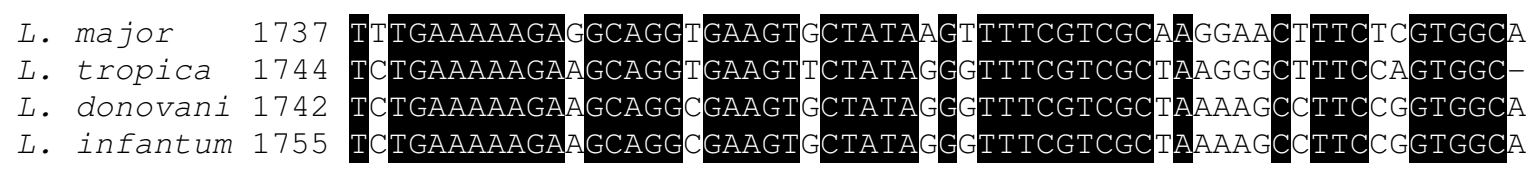
L. major
1797 AACGAACGGCAGCAAAAAAGGGAG
L. tropica
L. donovani 1802 AACAAACCGCAAAAAAAAGAGAG-
L. infantum 1815 AACAAACCGCAAAAAAAAGAGAG- 